\title{
Multisensor system for the protection of a critical harbour infrastructure
}

\author{
M. Kastek ${ }^{1}$, R. Dulski ${ }^{1}$, M. Życzkowski ${ }^{1}$, M. Szustakowski ${ }^{1}$, \\ P. Trzaskawka ${ }^{1}$, W. Ciurapiński ${ }^{1}$, G. Grelowska ${ }^{2}$, I. Gloza $^{2}$, \\ S. Milewski ${ }^{2} \&$ K. Listewnik ${ }^{2}$ \\ ${ }^{1}$ Institute of Optoelectronics, Military University of Technology, Poland \\ ${ }^{2}$ Polish Naval Academy, Navigation and Naval Weapons Division, \\ Poland
}

\begin{abstract}
The critical facilities within a harbour area require special protection, including security systems capable of monitoring both surface and underwater areas. The paper presents the concept of a multisensor security system for harbour protection, capable of complex monitoring of selected critical resources. The proposed system consists of a command centre and several different sensors deployed in key areas, providing effective protection from penetration from land and sea, with special attention focused on the monitoring of the underwater zone. The initial project of such systems was presented, its configuration and initial tests of the selected components. The protection of a surface area is based on medium-range radar, LLTV, and infrared cameras. The protection of an underwater zone is based on sonar, acoustic, and magnetic barriers. Both systems are combined into one, integrated multisensor monitoring system. Some results of theoretical analyses based on the detection of a fast and small surface were presented.
\end{abstract}

Keywords: security system, multispectral detection, data fusion.

\section{Introduction}

Geopolitical changes at the turn of the centuries had great impact on global security threats and challenges.

The danger of terrorist attacks exists also during peace time, and naval forces are not always ready to counter the unexpected terrorist attack. Such attacks may 
occur during the force withdrawal phase of peacekeeping operations, when the state of alertness is somehow lowered. The threat of terrorist attack rises as the crisis situation is being developed and in such crisis situation the sabotage and espionage acts are also likely to take place.

The attacks conducted by terrorists or other groups or individual persons against naval bases, harbours and other infrastructure can be accomplished in the following manner [1-3]:

- surface attack with manned and unmanned vessels,

- underwater attacks using divers, mini subs and explosives or sea mines,

- land attacks using bombs, handguns, grenades, mortars, etc.,

- aerial attacks with manned of unmanned crafts, balloons or kamikaze attacks,

- nuclear, biological and chemical agents attack,

- cybernetic attacks (breaking the security protocols, sending false alarm messages, stealing the data).

The European Union has introduced the Regulation $725 / 2004$ on enhancing ship and port facility security and the Directive $2005 / 65 / \mathrm{WE}$ on enhancing the port security. Security system for the protection of critical port infrastructure (e.g. gas or oil terminal) should conform to the requirements included in „Marine Terminal Physical Security" document, which describes the protection of oil terminals. The harbour security system is composed of several interacting components, like: Vessel Traffic System (VTS) and Automatic Identification System (AIS). Both systems mentioned above are now being integrated in single Port Management System (PMS) or Vessel Traffic Management and Information System (VTMIS). The main task of such systems is to assure safe traffic both in roadstead and in internal harbour zone. The land zone of the harbour should be also protected, including gates, fences and selected installations, like pipelines or tanks. As a result the security systems for the harbour protection include such solutions as:

- radar-camera system for the identification and tracking of personnel, land vehicles and surface vessels In order to provide perimeter protection, access control and visual monitoring of the area [4-6],

- active (sonar) and passive (magnetic barriers) systems to monitor the surface and underwater activity (including divers) [7].

\section{Concept of harbour security system}

The requirements on system architecture, functionality and instrumentation were formulated on the basis of broad analyses and studies, including knowing concepts of similar systems. The protected areas cover the terrain and critical land infrastructure, shore installations and selected sea zone.

There are many types of commercially available surveillance systems for the protection of such large-area objects like harbours [1]. However, for land surveillance they always utilize such components as:

- radar-visual observation systems with automatic target detection and tracking by a radar and then cueing the cameras for visual recognition, 
- CCTV paired with thermal cameras for day and night observation capability, - data and image fusion modules to obtain the highest possible effectiveness of the system,

- data visualization module to merge all useful for a system operator data and to visualize of the target trajectory on a digital map.

Above mentioned components, primary developed for land surveillance, were adapted to sea surface monitoring tasks. An appropriate implementation is a very important. For example, land radar-visual observation systems can produce significant noise in case of windy weather and rippled sea. As a result the noise filtration algorithms have to be implemented, both in radar and camera systems $[8,9]$.

Underwater and surface zones are monitored by passive and active systems. Such solutions (sonars and magnetic barriers) are used in restricted harbour areas, highly sensitive to unauthorized access (entrance to gas and oil terminals, pipelines, ships anchored at terminals) [3, 7]. The system with integrated passive and active sensors and the signal processing and fusion units should be able to detect and identify surface and underwater targets, including divers.

All subsystems used for harbour protection should be as autonomous as possible and deployed independently in the protected area. They should be connected via a secured cable or radio Ethernet network to obtain a complex, perimeter protection system. Such solution is suggested by the authors as a coherent system for the protection of port area, managed by a single command and control centre.

The illustration of this protection concept is shown in Fig. 1.
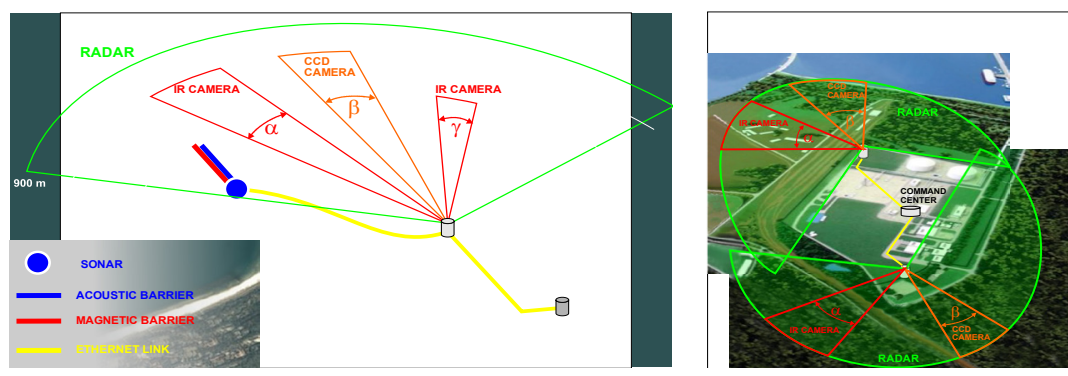

Figure 1: Sea (on the left) and land (on the right) zones protected by the multisensor system.

The perimeter line (fence) is remotely monitored and additionally the inside area is also under surveillance, including the restricted areas. Detailed requirements for radar-camera system are rather complex [10-13], but basic requirements are as follows:

- automatic broad-area surveillance,

- increased effectiveness by merging radar and day/night image data,

- detection and tracking of the intruder, visualization on a digital map with movement information (speed, direction) which add to the threat assessment by a system operator, 
- cost-effective solution, considering the size of the protected area, maintenance and running costs.

Automatic surface monitoring is assured by radar scanning in the full $\left(360^{\circ}\right)$ or selected horizontal angle in $24 / 7$ regime. System is continuously scanning the protected zones and triggers the alarm in case of intruder detection. As it was already mentioned, the sea environment generates significant amount of noise, and the radar signals have to be filtered in order to minimize false alarm rate. Currently the radars used in the security systems are modified versions of short range sea radars. They have ranges from $200 \mathrm{~m}$ to $3 \mathrm{~km}$ and work at the frequencies from $9 \mathrm{GHz}$ to $77 \mathrm{GHz}$, (millimetre radars). The signal processing hardware should have network interface to transmit radar data to target tracking system $[12,13]$. Software layer of a tracking system should provide:

- user-definable protected zones,

- rejection of background reflections (both land and sea background),

- definable number of reflections from target that initiate or stop tracking,

- sensitivity adjustment according to disturbance level,

- transfer the target data to the radar system processor.

The object (target) type is determined on the basis of radar track data and harbour AIS system. Surface vessels not identified by AIS are considered as threats, which triggers the alarm on the operator's console and cue the cameras automatically to target. The target is viewed simultaneously by daylight and thermal cameras. Both should have automatic focusing aided by the distance information provided by the radar. In order to obtain best possible image quality the image fusion is performed and the background clutter is filtered out [14-16]. The surface surveillance system should then provide: estimation of target movement and position reception of AIS data, target identification, rising the alarm and camera cueing, image filtering and fusion, and sending the final visual data to the operator's console.

The actual situation in the harbour area (sea and land) is presented on a digital map with marked infrastructure objects and protected, restricted zones. All detected objects are displayed (tracked by radar, recognized by AIS, unrecognized-threats) using the colour code. Data visualization system presents also video images of the tracked objects to aid the operator's decision on the course of action. The operator upon receiving the alarm signal is obliged to act according to the defined security procedures. If the operator is not reacting, the system should initiate threat countering actions automatically, sending the information to the patrol units in the protected area. The patrol units are equipped with GPS modules and their position is also visualized on a digital map. Apart from automatic mode, the operator should have the option to control the system manually.

Protection of harbour areas was usually focused at surface threats. There was, however, less attention paid to the underwater threats. Underwater environment, due to its complex nature, is a serious challenge to effective threat detection. Such factors as high disturbance level due to reflections of sonar signals, changing depth, temperature and transparency of water make the active sonar scanning of the harbour area extremely difficult. 
The security system used for the monitoring of the underwater area of the port should provide detection and identification of threats, motion tracking, and threat neutralization.

In order to accomplish the above goals the following sensor types can be applied in underwater monitoring (Fig. 2): specialized active sonars, underwater physical barriers, acoustic barriers, magnetic barriers, optical and chemical sensors.

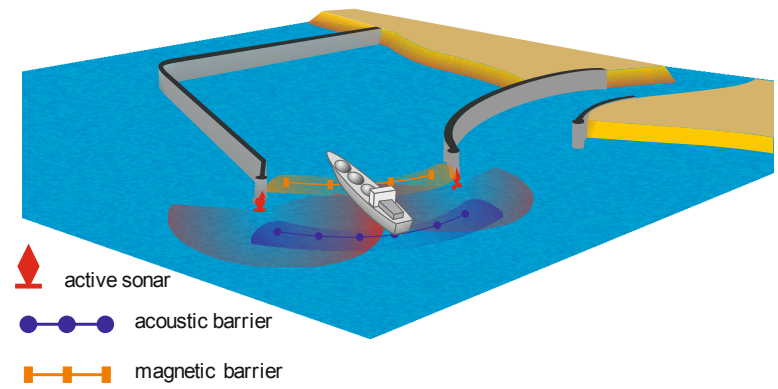

Figure 2: $\quad$ Components of underwater protection system for a harbour area.

The physical location of surface and underwater area monitoring systems is determined by the configuration of a protected area (Fig. 1). For example the radar-camera sensors (range 500-800 m) can be located on a single platform at the seaside or on breakwater. Location of underwater monitoring system is more complicated, because the barriers (acoustic and magnetic) must be placed at the seabed in the protected zone and the location of active sonar depends on the sea bottom configuration and required angle of observation. Both underwater systems operate on different principles, so they require separate signal processing units and command sub-centres for data processing and visualization. Main Command Centre should integrate the data from all the subsystems (subcentres) and it should be located in the Harbour Security Centre, which supervises all the security-related activities in the harbour area. The diagram describing functionality of the system including a command centre is presented in Fig. 3.

\section{Data fusion of security system}

Effective exploitation of all available information in all data channels requires the effective synthesis of sensor data to be applied [17-19]. Final effectiveness of a multi-sensor system should be (and usually is) better than that of a simple set of individual, independent sensors. The real benefit of multi-sensor setup is achieved only when the sensors provide information complementary to each other. Fig. 4 shows the diagram of data processing from all the system sensors from surface and underwater components. Both sub-systems have fully functional, separate data analysis modules, but the data fusion improves the target detection and lowers the false alarm rate. 


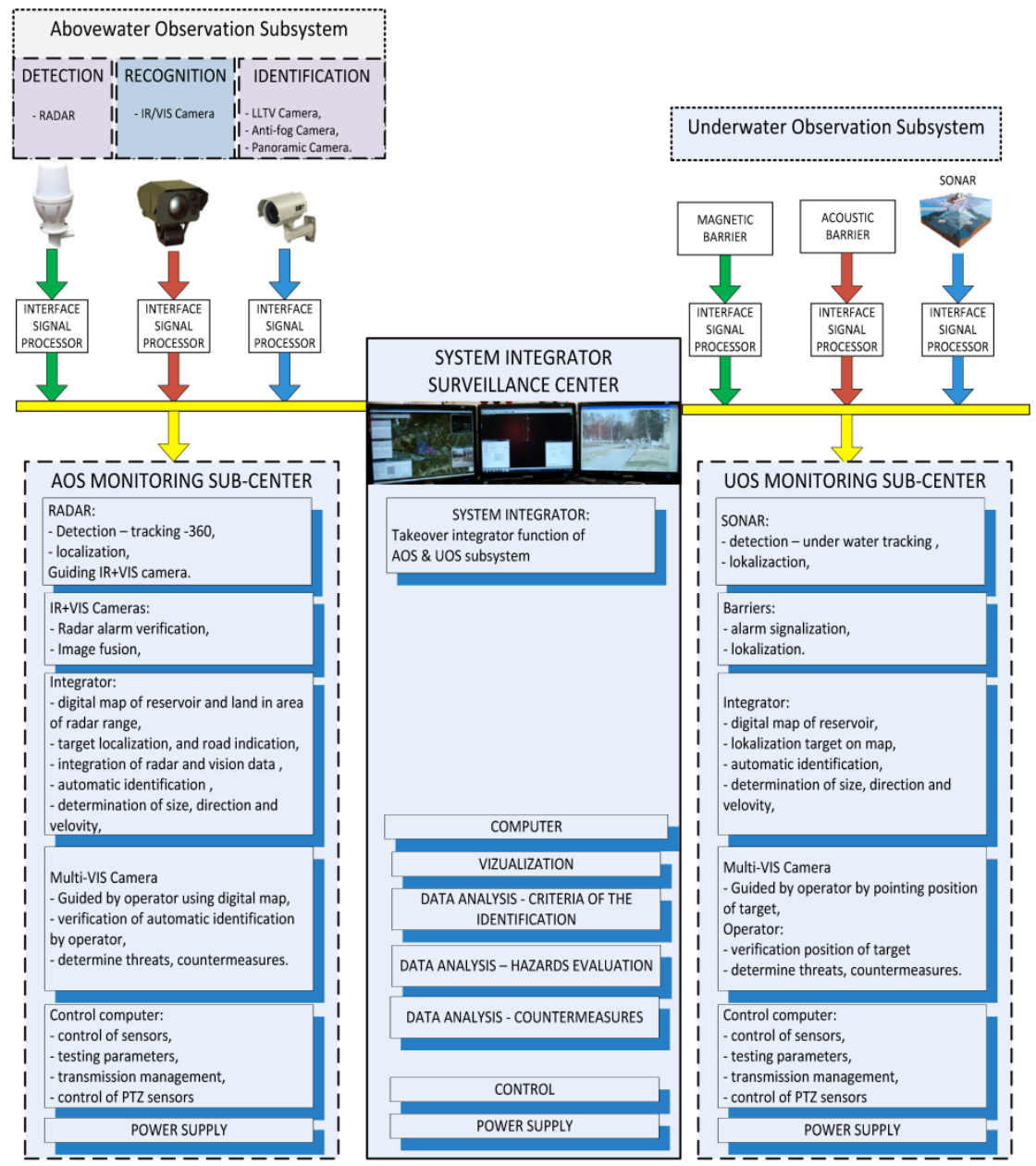

Figure 3: The diagram of functionality of the multisensor security system.

The application of a multi-sensor system diminishes the possibility of losing a target track due to loss of signal (e.g. when one sensor loses the sight of a target, others may still see it). Another example of complementary sensor operation can be observed due to different spectral bands and principles of operation of particular sensors. For example, radar sensor and IR camera have different properties (e.g. field of view, spectral band, spatial resolution). In a multi-sensor setup an omnidirectional sensor (radar) can be used to detect the target and then cue the high-resolution camera for final target identification. As it was already mentioned, the effectiveness of particular sensors depends on many factors, like weather conditions, background properties, distance and countermeasures used by an intruder. It may happen that single sensor has to operate in conditions far from optimal. The application of different sensors that are differently influenced 


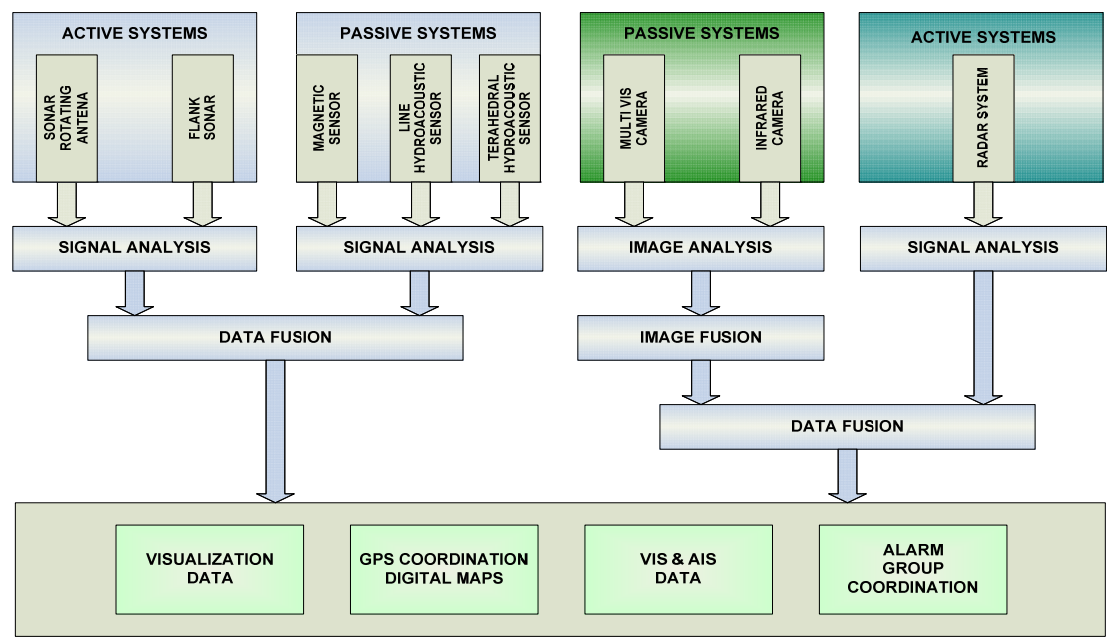

Figure 4: Data processing diagram in a multisensor security system for harbour protection.

by those external conditions assures constant proper system operation. Passive IR sensors (and VIS cameras) do not provide distance information, only angular dimensions can be extracted. The combination of radar sensor and a camera gives both the distance and target size data.

Data synthesis is a multi-layer process [20, 21]. In case of the perimeter protection system the initial data processing has to be performed and the target status has to be determined. The estimation of target status comprises of the characterization of an object itself and its movement. Object characterization is a process of data evaluation (not only sensor signals) that leads to complete target recognition: detection, direction, classification and identification. Detection is defined as confirmation of the presence of the target. Classification means that the detected target is assigned to one of the pre-determined classes of objects (e.g. human, boat, truck, ship). Identification level is achieved when the precise object description within its class can be made (human: armed assailant, boat: fishing cutter). Higher level of target discrimination imposes higher requirements on sensor resolution and signal-to-noise ratio at its output. The application of particular data synthesis algorithms in a multi-sensor security system depends on the overall system concept of operation. For example, object tracking relies usually on raw sensor data processing performed by a central system processing unit, whereas identification algorithms use complex data analysis at every functional level of the system. The general scheme of operation is presented in Fig. 5.

The presented concept of radar and camera data fusion is only one of many possible solutions. The same scheme is applied for underwater monitoring subsystem, where the passive and active sensor data are combined, processed and analysed. The Security system for the protection of selected critical infrastructure interacts (on the Command and Control centre level) with other security related 
systems of a seaport (like AIS) thus giving the operator full situational awareness in the vicinity of a protected zone.

Thermal and video cameras on the field measurement site and sample results registered during the tests of system cameras are shown in Fig. 6 and Fig. 7, respectively $[22,23]$.

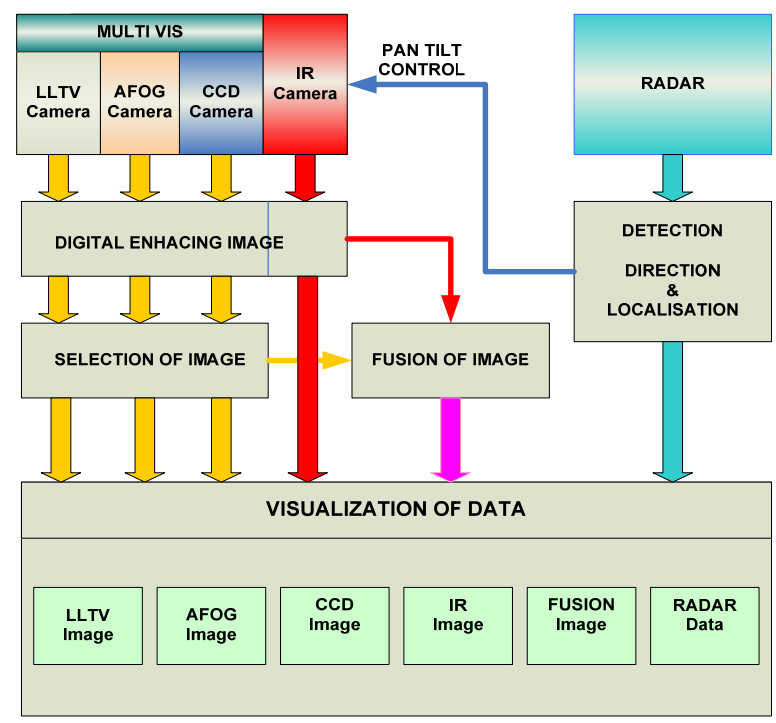

Figure 5: Concept of sensor data synthesis using radar, MultiVIS and IR cameras.
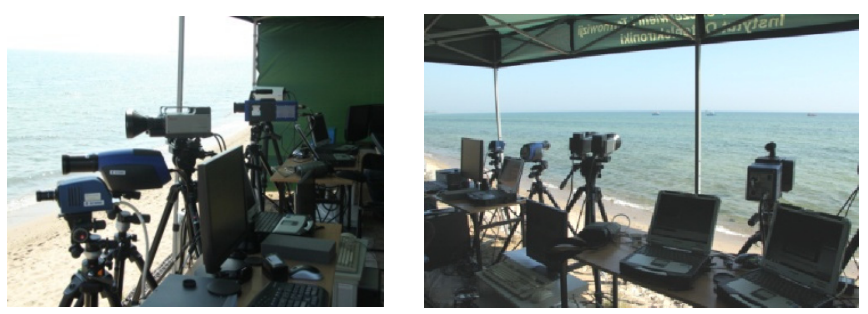

Figure 6: Thermal and video cameras on the field measurement site.
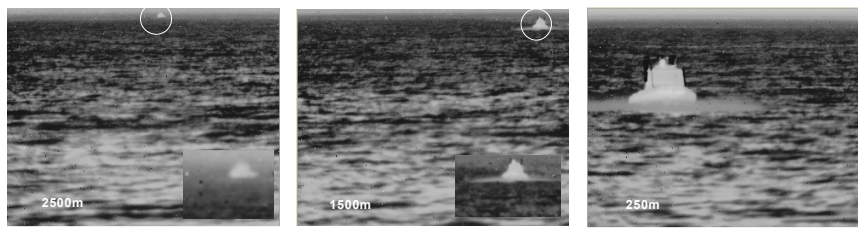

Figure 7: $\quad$ Sample thermal images of a RIB type motor boat while moving towards the camera (LWIR). 


\section{Conclusions}

The presented concept of a multisensor security system for the protection of critical infrastructure in the harbour area, comprising of underwater and surface monitoring sensors follows the current trends in contemporary security systems. The application of unique underwater sensor layout and the data fusion with surface sensors increases the probability of intruder detection in an attempt to violate the protected zone. Such solution is particularly important bearing in mind the threats and dangers of the present world.

Sensor data fusion demonstrated in the surface system components (daylight cameras, thermal cameras and radars) increases the probability of detection of small, fast surface objects like RIB boats, which are often used by terrorists in their attacks. The application of image enhancement techniques and fusion of thermal and daylight images may further increase the detection and visual identification range of small, fast-moving objects. The presented camera set-up and image processing algorithms were field tested with promising results, and the application of such camera configuration in security and surveillance systems is highly advisable.

The components of underwater monitoring systems were thoroughly tested in various weather conditions (including different states of the sea) at different harbour areas with different targets (ships, boats, divers). The tests were aimed at the evaluation of data analysis algorithms, estimation of detection probabilities and gathering additional data for signature database. The results were positive and the system configuration can be applied for the protection of critical infrastructure.

It can be finally stated that the presented multisensor system while deployed for the protection of harbour is very effective in detecting all kinds of objects that can be a threat to the harbour infrastructure.

\section{References}

[1] Suchman, D., Basic Do's and Don'ts of Designing, Installing and Operating a Harbor Protection system, Proceedings of TICA'05, 2005.

[2] Van Den Broek, A.C., Van Den Broek, S.P., Van Den Heuvel, J.C., Schwering, P.B.W., Van Heijningen, A.W.P., A multi-sensor scenario for coastal surveillance, Proc. of SPIE, 6736, 2007.

[3] Seibert, M., Rhodes, B.J., Bomberger, N.A., Beane, P.O., Sroka, J.J., Kogel, W., Kreamer, W., Stauffer, C., Kirschner, L., Chalom, E., Bosse, M., Tillson, R., SeeCoast port surveillance”, Proc. of SPIE 6204, 2006.

[4] Fasano, G., Forlenza, L., Accardo, D., Moccia, A., Rispoli, A., Integrated obstacle detection system based on radar and optical sensors, AIAA Infotech at Aerospace 2010, 3421, 2010.

[5] Baker C. J., Griffits H. D., Bistatic and Multistatic Radar Sensor for Homeland Security, www.natoasi.org/sensors2005/papers/baker.pdf.

[6] Butler, W., Poitevin, P., Bjornholt, J., Benefits of wide area intrusion detection systems using FMCW radar, Proc. of SPIE 6943, 2008. 
[7] Asada, A. , Kuramoto, K. , Tanaka, T., Oimatsu, K., Kawashima, Y., Nanri, M., Oyagi, T., Hantani, K., Development of underwater security sonar system, OCEANS 2006 - Asia Pacific, 4393879, 2007.

[8] Van Den Broek, S.P., Detection and classification of infrared decoys and small targets in a sea background, Proc. SPIE 4029, pp. 70-80, 2000.

[9] Dulski, R., Milewski, S., Kastek, M., Trzaskawka, P., Szustakowski, M., Ciurapiński, W., Zyczkowski, M., Detection of small surface vessels in near, medium and far infrared spectral bands, Proc. of SPIE 8185, 2011.

[10] Zyczkowski, M., Szustakowski, M., Kastek, M., Ciurapiński, W., Sosnowski, T., Module multisensor system for strategic objects protection, WIT Transactions on Information and Communication Technologies 42, pp. 123-132, 2009.

[11] Szustakowski, M., Ciurapinski, W.M., Zyczkowski, M., Palka, N., Kastek, M., Dulski, R., Bieszczad, G., Sosnowski, T., Multispectral system for perimeter protection of stationary and moving objects, Proc. of SPIE 7481, 2009.

[12] Zyczkowski, M., Palka, N., Trzcinski, T., Dulski, R., Kastek, M., Trzaskawka, P., Integrated radar-camera security system - Experimental results, Proc. of SPIE 8021, 2011.

[13] M. Kastek, R. Dulski, M. Życzkowski, M. Szustakowski, P. Trzaskawka, W. Ciurapinski, G. Grelowska, I. Gloza, S. Milewski and K. Listewnik, Multisensor system for the protection of critical infrastructure of a seaport, Proc. SPIE 8388, 2012.

[14] Dulski, R., Szustakowski, M., Kastek, M., Ciurapinski, W., Trzaskawka, P., Zyczkowski, M., Infrared uncooled cameras used in multi-sensor systems for perimeter protection, Proc. of SPIE, 7834, 2010.

[15] Kastek, M., Madura, H., Sosnowski, T., Passive infrared detector used for infrastructure protection, WIT Transactions on the Built Environment 108, pp. 61-70, 2009.

[16] J. Bareła, M. Kastek, K. Firmanty, P. Trzaskawka and R. Dulski, Determining detection, recognition, and identification ranges of thermal cameras on the basis of laboratory measurements and TTP model, Proc. SPIE 8355, 2012.

[17] Seastrand, M.J., Maritime microwave radar and electro-optical data fusion for homeland security, Proc. of SPIE, 5403 (PART 2), pp. 673-682, 2004.

[18] Hall, D. L., Llinas, J., An introduction to multisensor data fusion, Proc. IEEE vol. 85 no. 1, pp. 6-23, 1997.

[19] Klein, L. A., Sensor and Data Fusion Concepts and Applications, SPIE, 1993.

[20] Dulski, R., Kastek, M., Bieszczad, G., Trzaskawka, P., Ciurapiński, W., Data fusion used in multispectral system for critical protection, WIT Transactions on Information and Communication Technologies 42, pp. 165-172, 2009.

[21] Ciurapinski, W., Dulski, R., Kastek, M., Szustakowski, M., Bieszczad, G., Zyczkowski, M., Trzaskawka, P., Data fusion concept in multispectral 
system for perimeter protection of stationary and moving objects, Proc. of SPIE 7481, 2009.

[22] Dulski, R., Powalisz, P., Kastek, M., Trzaskawka, P., Enhancing image quality produced by IR cameras, Proc. of SPIE 7834, 2010.

[23] Kastek, M., Dulski, R., Zyczkowski, M., Szustakowski, M., Ciurapiński, W., Firmanty, K., Pałka, N., Bieszczad, G., Multisensor systems for security of critical infrastructures - Concept, data fusion, and experimental results, Proc. of SPIE 8193, 2011. 